\title{
Attachment cross-sections of protonated and deprotonated
}

\section{water clusters}

\author{
Sébastien Zamith ${ }^{1,2}$, Grégoire de Tournadre ${ }^{1,2, *}$, Pierre Labastie $^{1,2}$, and Jean-Marc L'Hermite ${ }^{1,2}$ \\ ${ }^{1}$ Université de Toulouse ; UPS ; Laboratoire Collisions Agrégats Réactivité, IRSAMC ; F-31062 \\ Toulouse, France \\ ${ }^{2}$ CNRS ; UMR 5589 ; F-31062 Toulouse, France
}

\begin{abstract}
Attachment cross-sections of water molecules onto size selected protonated $\left(\mathrm{H}_{2} \mathrm{O}\right)_{n} \mathrm{H}^{+}$ and deprotonated $\left(\mathrm{H}_{2} \mathrm{O}\right)_{n-1} \mathrm{OH}^{-}$water clusters have been measured in the size range $\mathrm{n}=30-140$ for $10 \mathrm{eV}$ kinetic energy of the clusters in the laboratory frame. Within our experimental accuracy, the attachment cross-sections are found to have the same magnitude and size dependence for both species. It is shown that electrostatic interactions are likely to play a role even for the largest sizes investigated.
\end{abstract}

\section{Introduction}

Charged particles play a crucial role in the nucleation of droplets in the atmosphere [1]. Due to electrostatic interactions, charged particles promote the formation of droplets. Since

* present address: Laboratoire de Recherche en Nanosciences, bâtiment 6, Université de Reims, 51687 Reims, France 
the cloud chamber experiment of Wilson back in 1897 [2], ions of different sign have been shown to induce significantly different nucleation rates $[3,4,5,6,7,8,9]$. Negatively charged water clusters seem to nucleate more efficiently than positively charged species; the origin of this sign preference for nucleation is still controversial $[10,11,12,13,14,15]$. Are higher attachment cross sections for anions than for cations responsible for this sign preference? The present paper brings a contribution to this issue. We compare the attachment crosssection of protonated $\left(\mathrm{H}_{2} \mathrm{O}\right)_{n} \mathrm{H}^{+}$and deprotonated $\left(\mathrm{H}_{2} \mathrm{O}\right)_{n-1} \mathrm{OH}^{-}$water clusters, for sizes ranging from $n=30$ to 140 . Attachment rate and cross-sections are measured for clusters colliding with water molecules at $10 \mathrm{eV}$ collision energy in the laboratory frame. We find the cross-sections to be essentially identical for anions and cations.

The paper is organized as follow: Section II gives a rapid overview of the experimental setup. Section III details how the cross-sections and rates are deduced from our measures and Section IV presents and discusses the experimental results. Conclusions are drawn in Section V.

\section{Experimental setup}

The experimental setup and procedure used here to measure attachment cross-sections of deprotonated water clusters are essentially the ones used in ref. 16. Briefly, both protonated and deprotonated water clusters are produced in a gas aggregation source. Depending on the polarity of the voltages used in the setup one can study either positively or negatively charged clusters. After their production, the clusters seeded in helium carrier gas are thermalized at $25 \mathrm{~K}$ in a heat bath. The clusters are then mass selected and slowed down to $10 \mathrm{eV}$ kinetic energy in the laboratory frame [17]. They enter a collision cell with a 
controlled pressure of water vapor. At the output of the collision cell, collision products are mass analyzed with a time of flight mass spectrometer. Our raw experimental data consist of time of flight mass spectra with one peak corresponding to the parent cluster and several additional peaks corresponding to the attachment of water molecules onto the parent cluster. We have shown in ref. 16 that no evaporation occurs after attachment and until the clusters reach the detector, in the present experimental conditions. As will be discussed below, absolute attachment rates or cross-sections can be directly deduced from such mass spectra.

In order to be able to compare as accurately as possible the cross-sections for protonated and deprotonated water clusters, measurements were performed for some sizes alternately for both species in the same runs, without changing anything in the setup but the polarity of the high voltages. The measurements were reproduced on different days under different source conditions and pressures in the cell. We thus achieve a much better precision for the measurement of the ratios of the cross sections of anions and cations than for the cross sections themselves.

\section{Attachment rate and cross-section}

From our measured mass spectra one can easily deduce the attachment rates or collision cross-sections. The attachment rate is simply given by: 


$$
k=-\frac{v_{\text {cluster }}}{\rho l} \ln \frac{I}{I_{0}}
$$

where $v_{\text {cluster }}$ is the cluster velocity in the laboratory frame, $\rho$ is the density in the cell and $l$ is the cell length. $I$ is the intensity of the peak of intact parent cluster and $I_{0}$ is the total intensity integrated over all the peaks.

The attachment rate is related to the cross section by:

$$
k=\int_{0}^{\infty} \sigma\left(v_{r e l}\right) v_{r e l} f\left(v_{r e l}\right) d v_{r e l}
$$

where [18]:

$$
f\left(v_{\text {rel }}\right)=\left(\frac{2 m}{\pi k_{B} T}\right)^{1 / 2} \frac{v_{\text {rel }}}{v_{\text {cluster }}} e^{-\frac{1}{2} m\left(v_{\text {cluster }}^{2}+v_{\text {rel }}^{2}\right) / k_{B} T} \sinh \left(\frac{m v_{\text {rel }} v_{\text {cluster }}}{k_{B} T}\right)
$$

is the distribution of relative velocities $v_{r e l}$ between the cluster and the colliding molecules. $T$ is the temperature of the cell, $k_{B}$ the Boltzmann constant and $m$ is the mass of the water molecule. Furthermore, if one assumes that the cross-section $\sigma\left(v_{r e l}\right)$ is a slowly varying function of $v_{r e l}$, the cross-section deduced from Eq. 1 and Eq. 2 is:

$$
\sigma=-\frac{v_{\text {cluster }}}{\overline{v_{\text {rel }}}} \frac{\ln \left(I / I_{0}\right)}{\rho l}
$$

where $\overline{v_{r e l}}$ is the average relative velocity given by [18]:

$$
\overline{v_{\text {rel }}}=v_{\text {cluster }}\left(\operatorname{erf}(\sqrt{a})+\frac{1}{2 a} \operatorname{erf}(\sqrt{a})+\frac{e^{-a}}{\sqrt{\pi a}}\right)
$$

with $a=\frac{m v_{\text {cluster }}^{2}}{2 k_{B} T}$. 
In the following both the attachment rates and cross-sections will be presented. It is advantageous to compare directly the experimental attachment rates to theoretical ones since no assumption has to be made about the cross-section.

\section{Experimental Results and Discussion}

In Figure 1 are presented the measured attachment cross-sections for protonated and deprotonated water clusters, and Figure 2 shows the corresponding attachment rates.

At first we should mention that compared to our previous results $[16,19]$ we find crosssections roughly $30 \%$ higher than previously measured. A slightly different, more accurate data processing has been performed and it turned out that results of ref. 16 should be multiplied by a factor 1.3. The conclusions of ref. 16 remain the same however: the model presented in ref. 16 still reproduces satisfactorily our results.

Fig. 1 and Fig. 2 show that the attachment cross sections and the attachment rates, respectively, of protonated water clusters are close to those measured for deprotonated clusters. As expected, cross-sections and attachment rates increase with the cluster size. The attachment probabilities of protonated clusters seem to be slightly lower than those of deprotonated clusters at small sizes, but this trend is not marked enough, within our experimental accuracy, to draw a definite conclusion about a possible charge effect. In any case, the tiny difference that might exist between the attachment probabilities of cations and anions discard higher attachment cross sections of anions as a possible cause of the sign preference for nucleation (in the frame of classical nucleation theory indeed, the nucleation rate depends only weakly on the attachment cross section $[20,21])$. 
Before analyzing the effect of electrostatic interactions between the impinging molecule and the cluster, we will first compare our experimental results with the simplest model, considering the clusters and the water molecules as hard-spheres. The geometric crosssection is given by [22]:

$$
\sigma_{g e o}=\pi\left(R_{c}+r_{m}\right)^{2}
$$

where $R_{c}$ is the cluster radius and $r_{m}$ is the molecular radius. The rate is then:

$$
k_{g e o}=\sigma_{g e o} \overline{v_{r e l}}
$$

It is difficult to have a reliable estimate of both the molecular and cluster radius. One can assume that the clusters have the same density as the bulk and deduce the radius of a cluster of size $n$ as $R_{c}(n)=\sqrt[3]{\frac{3 n m}{4 \pi \rho}}=n^{1 / 3} r_{m}$. The density $\rho$ can be taken to be the density of hexagonal ice, liquid water or amorphous ice for instance. These three densities yield values for $r_{m}$ in the range 1.93-1.98 $\AA$. However, clusters might have densities differing from the bulk values. In the size range considered here, clusters are expected to be amorphous $[23,24]$. In ref. [24], the authors deduce from theoretical calculations a diameter of $2.7 \mathrm{~nm}$ for a cluster containing 293 molecules. This yields a value $r_{m}=2 \AA$. Furthermore from the distribution of molecular distances from the cluster center of mass (figure 24 of ref. 24), one can deduce values for the molecular radius around $1.9-2.0 \AA$ in the size range $n=22-931$. From our measurements, if we assume that for the bigger sizes the attachment cross-section is given by the hard-sphere model, we deduce a molecular radius of $2.28 \AA$ (red line in figure 1).

Recently Lengyel et al [25] have measured attachment cross-sections for neutral water clusters. For an average size of $n=260$, they have found a value $\left(955 \AA^{2}\right)$ very similar to the 
one measured in our group for protonated water clusters (980 $\AA^{2}$ for $n=250$ [16]) for similar collision energies. However in [25] the authors claim that they observe a cross-section which is about 2.5 times bigger than the geometric cross-section, taking a value of $1.77 \AA$ for $r_{m}$ and calculating the geometric cross-section as $\sigma_{g e o}=\pi n^{2 / 3} r_{m}^{2}$. The corresponding geometric cross-section is plotted as a dotted line in Fig. 1. The correct definition for the geometric cross-section should be the one given by Eq. 6 and we believe that the $r_{m}$ value taken by Lengyel et $a$ is probably a low estimate.

In reference [25] the collisions occur between neutrals whereas we studied ion-neutral collisions: One should therefore expect higher cross-sections in our experiment, due to iondipole and ion-induced dipole attractive interactions. The fact that similar cross-sections are found in both experiments, for neutral and charges species, would tend to indicate that, in this size range and this collision energy range, electrostatic interactions do not play a major role. In this case, provided that clusters are approximately spherical, the attachment crosssections can be approximated to a good accuracy by hard sphere cross-sections.

However, Lengyel et al observe a variation of the cross-section when using different collision partners. These observations are rather in favor of a cross-section still dominated by long range interactions. On the other hand in $[16,19]$ we observed that as the size increases, the cross-section no longer depend on the collision energy. This observation is in favor of our argument that the cross-section becomes dominated by the geometric size of the clusters for the higher sizes.

In order to evaluate the enhancement of the cross-sections (or the rates) due to electrostatic interactions, we calculated these cross sections in the frame of a Langevin 
model, using the average dipole orientation (ADO) theory [26]. In this approach the interaction between the charged cluster and the neutral molecule is given by:

$$
V(r)=-\frac{\alpha}{2 r^{4}}-\frac{c_{l} \mu_{D}}{E_{c}}+\frac{E_{c} b^{2}}{r^{2}}
$$

The first term represents the charge-induced dipole interaction, the second term is the charge-permanent dipole interaction, and the third term is the centrifugal barrier. $r$ is the distance between the center of the cluster and the molecule, $E_{c}$ is the collision energy in the center of mass frame and $b$ is the impact parameter. The polarizability of water is $\alpha=9.93$ a.u. [27] and the dipole moment of water $\mu_{D}$ equals 0.73 a.u. [28]. $C_{l}$ is the dipole locking constant introduced by Su et al [26] in ADO theory. In order to introduce size effects, we used the models developed by Kummerlöwe et al [29]. In this paper two models are considered: the hard sphere average dipole orientation (HSA) and the surface charge capture (SCC) model. In the SCC model the charge is mobile at the surface of the cluster whereas in the HSA model it is localized at the center of the cluster. Those two models give lower and upper bounds for the effect of the electrostatic interactions, which are likely to be underestimated in the HSA model and overestimated in the SCC model. The attachment rate in the frame of these models is calculated as:

$$
k_{S C C, H S A}=\int_{0}^{\infty} \sigma_{S C C, H S A} f\left(v_{r e l}\right) v_{r e l} d v_{r e l}
$$

where the expressions for $\sigma_{S C C, H S A}$ are given in ref. 29. The rates obtained from a numerical integration of Eq. 9 are plotted together with the experimental rates in figure 2. The theoretical rates have been calculated assuming a molecular radius of $1.98 \AA$ and a dipole locking constant $C_{l}=0.215$ [26]. For large sizes, the overall result of these 
calculations are not in contradiction with our experimental observations, since experimental attachment rates fall in between the predictions of the HSA and the SCC models, which are expected to underestimate and overestimate, respectively, the electrostatic interactions thus the attachment rates. But none of those two models can explain our observed variation of the attachment rates with size. The only way we have found so far to reproduce our results is to invoke dynamical effects [16]: when the collision duration becomes shorter than the time required for redistributing the collision energy among the internal degrees of freedom of the cluster, the attachment probability is reduced.

Our experimental cross-sections are about 1.3-1.4 higher than the geometric crosssections assuming $r_{m}=1.98 \AA$. So electrostatic interactions probably still play a role even at the largest cluster sizes investigated here. However, assuming a slightly bigger molecular radius would reduce the deduced role of electrostatic interactions. Finally, despite our effort to measure reliable cross-sections, it is hard to completely dismiss the possibility that the pressure reading is off by an amount that would suffice to explain the deviation from the hard sphere cross-section at large sizes.

The modeling of particles growth requires the knowledge of attachment cross-sections $[20,21]$. Concerning water nucleation in the atmosphere, the cross sections of the particles is often estimated in the frame of the hard sphere model using the ice density [30]. Our measures indicate that more realistic cross-sections should be used. However, these cross sections cannot be directly deduced from the present work since our measurements are not made at temperatures usually present in the atmosphere. Indeed, our lowest collision energy in the present study corresponds to mean kinetic energy of water molecules at a temperature of about $900 \mathrm{~K}$. Nevertheless, we can try to extrapolate our results to small 
cluster sizes at room temperature. To do so, we assume that the cross-section is given by either the HSA or SCC model multiplied by a factor due to dynamical effects, namely $\left(1-e^{-\frac{\tau_{c}-\tau_{0}}{\tau_{v}}}\right)$ (see ref. 16). Fig. 3 presents the ratio of cross-sections calculated in this way to geometric cross-section. This crude model predicts an enhancement of the cross-section compared to the geometric cross-section, with a maximum for sizes around 10 . As the size increases, the cross-section is a factor in between 1.2 and 2 larger than the geometric crosssection. Quite surprisingly, the size dependence of the calculated cross sections does not strongly depart, except for very small clusters, from geometric cross sections. This behavior is due to two conjugate effects that almost counterbalance: on the one hand, the electrostatic enhancement of the cross sections at low energy, on the other hand their reduction by the dynamical effect presented in reference [19].

\section{Conclusion}

We have made a direct comparison of attachment cross-sections for protonated and deprotonated water clusters. Within our experimental conditions, we observe no significant effect of the sign of the charge of the clusters. The predominant effect is due to previously invoked dynamical effects $[16,19]$. At large sizes, the attachment cross sections are higher than geometric cross sections, which seems to indicate that electrostatic interactions enhance the attachment probability. On the other hand, our cross sections being close to the ones obtained for neutral species by J. Lengyel and coworkers [25] suggests that electrostatic interactions do not play a major role in the attachment process. This contradiction could not be removed by analyzing our results in the frame of a Langevin 
model; clearly, the problem of the attachment of water molecules onto water clusters still needs further investigations. 


\section{references}

[1] A. Hirsikko, T. Nieminen, S. Gagné, K. Lehtipalo, H. E. Manninen, M. Ehn, U. Hõrrak, V.-M. Kerminen, L. Laakso, P. H. McMurry, A. Mirme, S. Mirme, T. Petäjä, H. Tammet, V. Vakkari, M. Vana, and M. Kulmala, Atmos. Chem. Phys., 11, 767 (2011)

[2] C. T. R. Wilson, Philos. Trans. R. Soc. London, Ser. A 189, 265 (1897)

[3] L. B. Loeb, A. F. Kip, and A. W. Einarsson, J. Chem. Phys. 6, 264 (1938)

[4] A. W. Castleman and I. N. TangJ. Chem. Phys. 57, 3629 (1972)

[5] H. Rabeony and P. Mirabel, J. Phys. Chem. 91, 1815-1818 (1987)

[6] M. Adachi, K. Okuyama, and J. H. Seinfeld, J. Aerosol Sci. 23, 327 (1992)

[7] T. Seto, K. Okuyama, L. de Juan and J. F. de la Mora, J. Chem. Phys. 107, 1576 (1997)

[8] K. Nagato, C. S. Kimb, M. Adachic, K. Okuyama, J. Aerosol Sci. 36, 1036 (2005)

[9] C.-C. Chen and H.-C. Cheng, J. Chem. Phys. 126, 034701 (2007)

[10] K. J. Oh, G. T. Gao, and X. C. Zeng, Phys. Rev. Lett. 86, 5080 (2001)

[11] V. B. Warshavsky and X. C. Zeng, Phys. Rev. Lett. 89, 246104 (2002)

[12] S. M. Kathmann, G. K. Schenter, and B. C. Garrett, Phys. Rev. Lett. 94, 116104 (2005)

[13] A. B. Nadykto, A. Al Natsheh, Fangqun Yu, K.V. Mikkelsen and J. Ruuskanen, Phys. Rev. Lett., 96, 125701 (2006)

[14] S. M. Kathmann, G. K. Schenter, and B. C. Garrett, Phys. Rev. Lett. 98, 109603 (2007)

[15] A. B. Nadykto, A. Al Natsheh, K. V. Mikkelsen, F. Yu, and J. Ruuskanen, Phys. Rev. Lett. 98, 109604 (2007)

[16] S. Zamith, P. Feiden, P. Labastie and J.-M. L'Hermite, J. Chem. Phys. 133, 154305 (2010)

[17] F. Chirot and S. Zamith and P. Labastie and J.-M. L'Hermite, Rev. Sci. Instrum. 77, 063108 (2006)

[18] S. Chapman and T. G. Cowling, The mathematical theory of non-uniform gases, Cambridge University Press, 3rd edition, 1970

[19] S. Zamith, P. Feiden, P. Labastie and J.-M. L'Hermite, Phys. Rev. Lett. 104, 103401 (2010)

[20] I. J. Ford, Phys. Rev. E 56, 5615 (1997)

[21] D. W. Oxtoby, J. Phys.: Condens. Matter 4, 7627 (1992)

[22] R. D. Levine and R. B. Bernstein, Molecular Reaction Dynamics and Chemical Reactivity (Oxford University Press, New York, 1987), p. 31-32 
[23] G. Torchet, J. Farges, M. F. de Feraudy, and B. Raoult, Ann. Phys. Fr. 14, 245 (1989)

[24] V. Buch, S. Bauerecker, J. P. Devlin, U. Buck and J. C. Kazimirski, Int. Rev. Phys. Chem. 23, 375 (2004)

[25] J. Lengyel, J. Kočišek, V. Poterya, A. Pysanenko, P. Svrčková, M. Fárník, D. K. Zaouris and J. Fedor, J. Chem. Phys. 137, 034304 (2012)

[26] T. Su, E. C. F. Su, and M. T. Bowers, J. Chem. Phys. 69, 2243 (1978)

[27] E. V. Tsiper, Phys. Rev. Lett. 94, 013204 (2005)

[28] S. L. Shostak, W. L. Ebenstein, and J. S. Muenter, J. Chem. Phys. 94, 5875 (1991)

[29] G. Kummerlöwe and M. K. Beyer, Int. J. Mass. Spectrom. 244, 84 (2005)

[30] J. Wölk, R. Strey, C. H. Heath, and B. E. Wyslouzil, J. Chem. Phys. 117, 4954 (2002) 


\section{Figure captions}

\section{Figure 1: (color online)}

Log-log plot of the experimental cross-sections for protonated (red triangles) and deprotonated (blue squares) water clusters. The clusters kinetic energy is $10 \mathrm{eV}$ in the laboratory frame. The full and dashed lines correspond to the hard-sphere cross-section given by Eq. 6 for $r_{m}=2.28 \AA$ and $1.98 \AA$, respectively. The dotted line is the plot of $\sigma_{g e o}=\pi n^{2 / 3} r_{m}^{2}$ for $r_{m}=1.77 \AA$.

\section{Figure 2: (color online)}

Experimental attachment rates (see equation 1 for the definition of the rates) for protonated (red triangles) and deprotonated (blue squares) water clusters. Theoretical rates are also plotted: hardsphere (full line), SCC (dashed line) and HSA (dotted line).

\section{Figure 3: (color online)}

Ratios of calculated attachment cross-section to geometric cross-section. The attachment crosssection is calculated using the HSA (dashed line) and SCC (full line) cross-sections and by taking into account dynamical effects. 


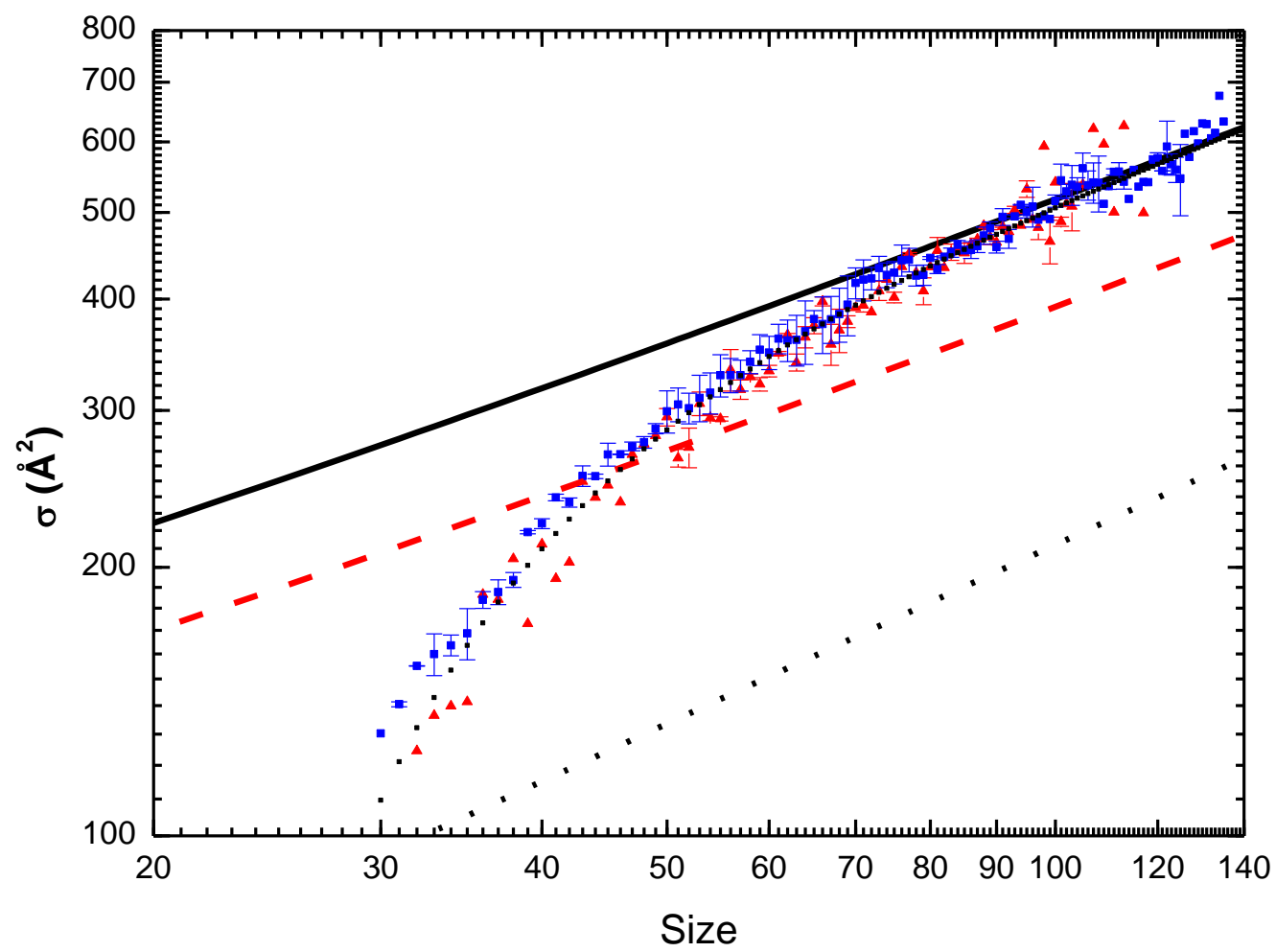

Figure 1 


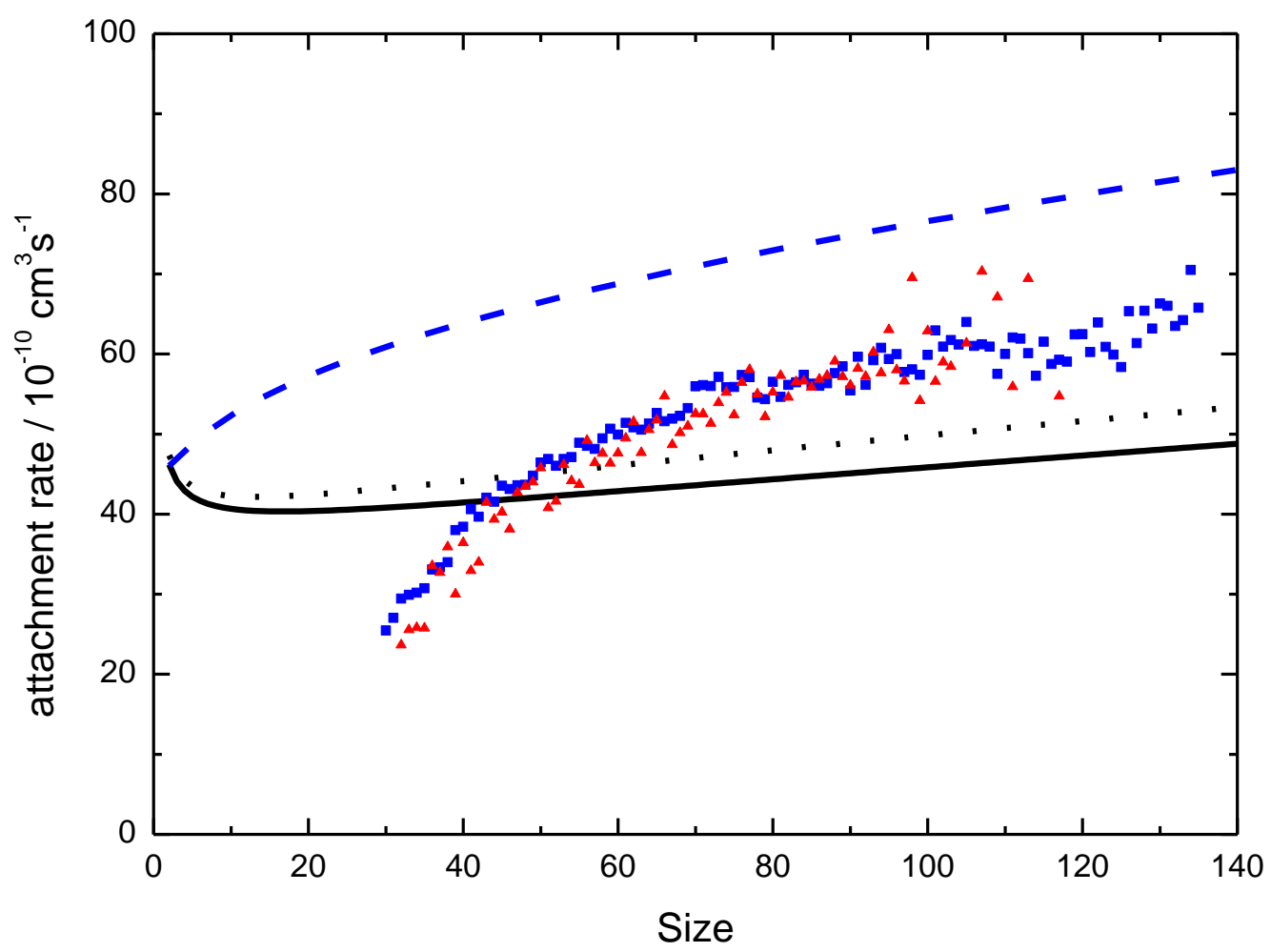

Figure 2 


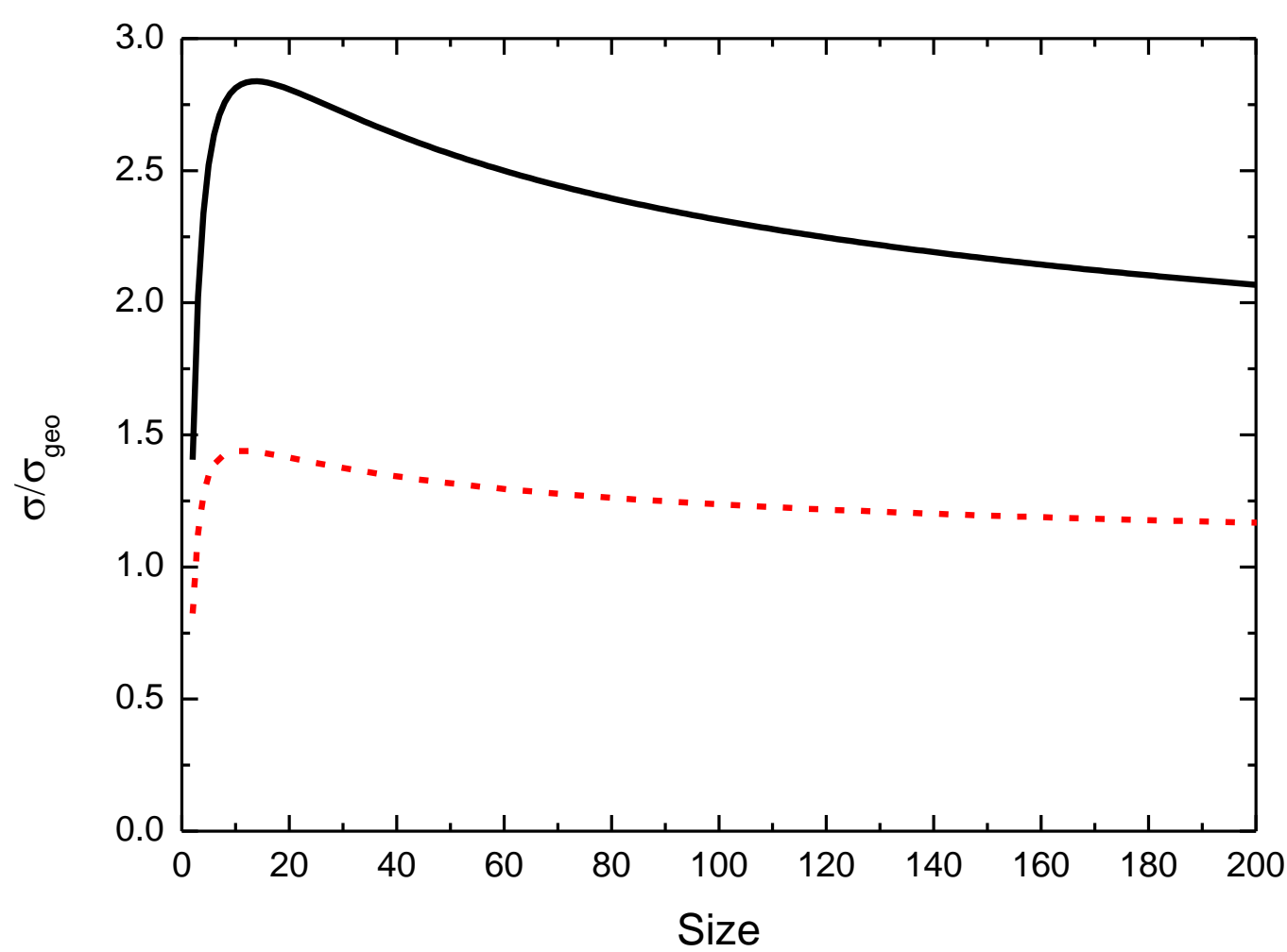

Figure 3 